\title{
MIMO Over-The-Air Research, Development, and Testing
}

\author{
Moray Rumney, ${ }^{1}$ Ryan Pirkl, ${ }^{2}$ Markus Herrmann Landmann, ${ }^{3}$ \\ and David A. Sanchez-Hernandez ${ }^{4}$ \\ ${ }^{1}$ Agilent Technologies, Edinburgh EH12 9DJ, UK \\ ${ }^{2}$ National Institute of Standards and Technology, Boulder, CO 80305-3337, USA \\ ${ }^{3}$ Fraunhofer Institute for Integrated Circuits (IIS), 91058 Erlangen, Germany \\ ${ }^{4}$ Universidad Politecnica de Cartagena, GIMRE, Antiguo Cuartel de Antigones, 30202 Cartagena, Spain \\ Correspondence should be addressed to David A. Sanchez-Hernandez, david.sanchez@upct.es
}

Received 9 May 2012; Accepted 9 May 2012

Copyright ( 2012 Moray Rumney et al. This is an open access article distributed under the Creative Commons Attribution License, which permits unrestricted use, distribution, and reproduction in any medium, provided the original work is properly cited.

\section{Introduction to the Special Issue}

Multiple-input multiple-output (MIMO) over-the-air (OTA) measurements and simulations for network and terminal performance evaluation and prediction have become very important research topics in recent years. Research into MIMO OTA for standardisation purposes has been ongoing in The Wireless Association (CTIA), the Third Generation Partnership Project (3GPP), and the European Cooperation in Science and Technology (COST) for three years. This is motivated by the urgent need to develop accurate, realistic, and cost-effective test standards for UMTS and LTE systems. Although many MIMO-capable networks are already deployed, there is pressure to finish the test standards by the end of 2012. While the first MIMO devices appeared some years ago and were commercially deployed two years ago, there are not yet any standards for testing MIMO performance OTA. The development of MIMO OTA test standards has proven to be particularly complex compared to single-input single-output (SISO) OTA, and developing a test standard is taking considerable time. Unlike SISO OTA, which was relatively straightforward and purely a function of the device, MIMO OTA is highly dependent on the interaction between the propagation characteristics of the radio channel and the receive antennas of the UE. Consequently, the existing SISO measurement techniques are unable to test the UE's MIMO properties. Many different MIMO test methods have been proposed, which vary widely in their propagation channel characteristics, size, and cost. Many challenges remain in the areas of identifying the optimal channel models and test $\operatorname{method}(\mathrm{s})$, and it is possible that the outcome could be that more than one test methodology will be standardized. Current standards activities are concentrated on showing if the proposed test methodologies provide the same results, with the ultimate goal being to clearly differentiate good from bad MIMO devices. The aim of this special issue, guest edited by a balanced representation from across academia and industry is to provide a valuable source of information for the state of this important research area.

Section 2 of this introductory paper provides an introduction to MIMO OTA standardization activities, and Section 3 describes the different test methodologies under consideration by $3 \mathrm{GPP} / \mathrm{CTIA}$. A comparison between test methodologies is made in Section 4. A summary of the papers accepted for publication in this special issue is presented in Section 5. These articles discuss important aspects of MIMO OTA testing and the latest advances of all test methodologies. The research represents the latest thinking of well-known experts in industry and academia and will undoubtedly influence future decisions on testing standardization. Some conclusions and future work are provided in Section 6.

\section{MIMO OTA Standardization Activities}

The work to standardize MIMO OTA measurement methods and performance requirements has evolved from the SISO OTA standards developed by CTIA in 2001 and later by 3GPP. Significant research in support of these standards was provided by COST actions 259 [1] and 273 [2]. Two figures of merit were chosen for SISO OTA: Total Radiated Power 
(TRP) and Total Reference Sensitivity (TRS), also known within CTIA as Total Isotropic Sensitivity (TIS). The TRP metric is calculated by computing the average of the radiated power over a sphere centred on the device under test (DUT). The TRS metric is the average over the same sphere of the minimum received power to achieve a particular bit error rate. This DUT receiver measurement is made while the DUT is transmitting at maximum power so that any radiated effects that might cause self-blocking or desensitization of the DUT receiver are fully captured.

The first CTIA SISO OTA specification [3] defined the TRP and TRS measurement methods to be made in an anechoic chamber using a reference antenna in two orthogonal polarizations. This method was also adopted by $3 \mathrm{GPP}$ in [4], which additionally specified device performance requirements. An alternative test method using a reverberation chamber was also specified in [4]. This was possible since it was shown empirically that the results obtained by averaging many individual measurements using a point source within an anechoic chamber were very similar to results achieved by averaging in a mode-stirred reverberation chamber, which over a suitable period of time will generate an isotropic field.

With the introduction of MIMO technology, and in particular spatial multiplexing, the methods developed for SISO OTA could not be directly used to measure the performance of MIMO devices. This led in late 2007 to the formation of a reverberation chamber subgroup within CTIA to study the feasibility of extending reverberation chambers for MIMO device testing. In April 2009, CTIA added an anechoic chamber subgroup to study the development of MIMO measurements in anechoic chambers. In 2009, the study of MIMO OTA was added to COST action 2100 and in March 2009, 3GPP approved the study item "Measurement of radiated performance for MIMO and multi-antenna reception for HSPA and LTE terminals" in [5]. In March 2011, the two CTIA groups were merged to create the MIMO OTA subgroup (MOSG), and finally, in February 2012, 3GPP approved the work item "Verification of radiated multi-antenna reception performance of UEs in LTE/UMTS" [6] to create a formal test specification for MIMO OTA.

The fundamental difference between SISO and MIMO performance is the radio propagation channel. For SISO, the DUT performance is independent of the channel, which is defined as isotropic and with no channel fading. The isotropy is achieved in the anechoic chamber by averaging many measurements made from a single angle of arrival/departure and in the reverberation chamber by the time domain averaging of the random angles of arrival/departure caused by the mode stirring.

An isotropic environment is necessary to evaluate TRP and TIS but is totally unsuited for evaluating the spatial multiplexing performance of MIMO devices. In order for spatial multiplexing to show any gain over SISO, operation requires that the signals received by each DUT antenna be sufficiently different that the DUT receiver can decode the individual data streams. In an isotropic environment, the signals received by each antenna will be the same and no spatial multiplexing gain will be possible. Thus to evaluate spatial multiplexing performance, it is necessary to subject the DUT to a nonuniform field through a combination of varying the angular spread of the signal or its polarization or a combination of both. When this nonuniform field is received by a DUT, which has nonidentical antennas then the possibility of spatial multiplexing gain becomes non zero.

Consequently, the two main challenges in evaluating MIMO devices are first to define the channel conditions (including any noise or interference), in which the performance is to be evaluated and then to physically create that environment and test the device. The latter step represents a new challenge to the test industry because until now, the emulation of channel propagation conditions has been done electrically using a channel emulator with a cabled (galvanic) connection to the DUT. This is the traditional method for measuring receiver performance but clearly bypasses the DUT's antennas and has no relevance to OTA testing. Thus, for MIMO OTA testing, the challenge of channel emulation has moved from the conducted domain to the radiated domain. This is a nontrivial problem that has been the subject of most of the industry research.

\section{3GPP Candidate Test Methodologies}

As a result of the 3GPP study item, seven different test methods have been proposed to 3GPP in Technical Report 37.976 [7] for creating the necessary environment to test MIMO performance. The test methods fall into two main groups: five based on anechoic chambers and two based on reverberation chambers.

Anechoic chamber candidate methods are as follows:

(A1) multiprobe method (arbitrary number and position),

(A2) ring of probes method (symmetrical),

(A3) two-stage method,

(A4) two channel method,

(A5) spatial fading emulator method.

Reverberation chamber candidate methods are as follows:

(R1) basic or cascaded reverberation chamber,

(R2) reverberation chamber with channel emulator.

The anechoic and reverberation methods take fundamentally different approaches towards achieving the same end goal- the creation of a spatially diverse radio channel. In the case of the anechoic chamber, multiple probes are used to launch signals at the DUT in order to create known angles of arrival, which map onto the required channel spatial model. This is a powerful approach although in order to achieve arbitrary channel model flexibility, large numbers of probes are required, which is costly and challenging to calibrate due to issues like backscatter. In the reverberation chamber method, the spatial richness is provided in 3D by relying on the natural reflections within the chamber, which are further randomized by use of mode stirrers that oscillate to provide a spatial field, which over long periods of time approaches an isotropic field. However, the instantaneous spatial field is not 




FIGURE 1: Multiprobe configuration (TR 37.976 [7] Figure 6.3.1.1-2).

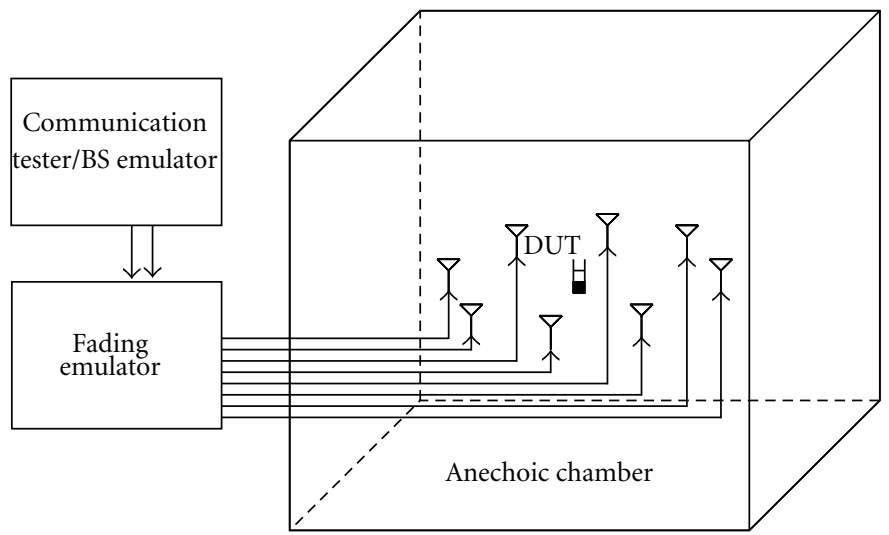

Figure 2: Example of MIMO/Multiantenna OTA test setup (TR 37.976 [7] Figure 6.3.1.2-1).

isotropic, which means that the reverberation chamber can be used to measure spatial multiplexing gain in decorrelated antennas. Each method will now be briefly introduced. For further details, refer to TR 37.976 [7].

3.1. A1 Multiprobe Method. The principle behind this method shown in Figure 1 is to create the desired channel model by positioning an arbitrary number of probe antennas in arbitrary positions within the anechoic chamber equidistant from the DUT, each antenna being faded by a channel emulator to provide the desired temporal component. By careful choice of the number and position of the probe antennas, it is possible to construct an arbitrarily complex radio propagation environment. The method is the most conceptually simple since there is a direct relationship between the required angular spread of the channel and the physical location of the probes.

A simple single cluster channel model with a narrow angular spread can be emulated using four antennas in a relatively small anechoic chamber with the DUT at one end and the probes at the other. More complex multicluster conditions can be generated with an increased number of probes and the transition to a larger anechoic chamber with the DUT placed in the centre and the probes on the perimeter of the chamber. The simplest configurations would locate the probes in the same azimuth plane to create a $2 \mathrm{D}$ environment. More complex 3D fields can be created by locating antennas on a different plane. The direct relationship between the probe antenna positions and the emulated channel model mean that in order to test the DUT from all angles, the DUT must be mounted on a rotating and tilting platform.

3.2. A2 Ring of Probes Method. The ring of probes method is based on a symmetric ring of probe antennas equidistant around the DUT, which is placed at the centre of the anechoic chamber as shown in Figure 2. As with the multiprobe method, each probe is fed by a channel emulator to generate the temporal characteristics of the desired channel model. Where the symmetrical ring of probes method differs however is that there is no longer a fixed relationship between the probe antenna positions and the angle of departure. Instead, the spatial components of the channel model are mapped onto the equally spaced probe antennas in such a way that an arbitrary number of clusters with associated angular spreads can be generated. This more flexible approach enables any 2D spatial channel model without having to reposition (and recalibrate) the probe antennas.

The number of antennas in the ring affects the accuracy with which the spatial dimension of the channel model can be implemented. A typical configuration is a 22.5 degree raster with vertical and horizontal polarization at each location giving a total of 32 probes each independently driven by a channel emulator.

3.3. A3 Two-Stage Method. The two-stage method takes a fundamentally different approach to creating the necessary 


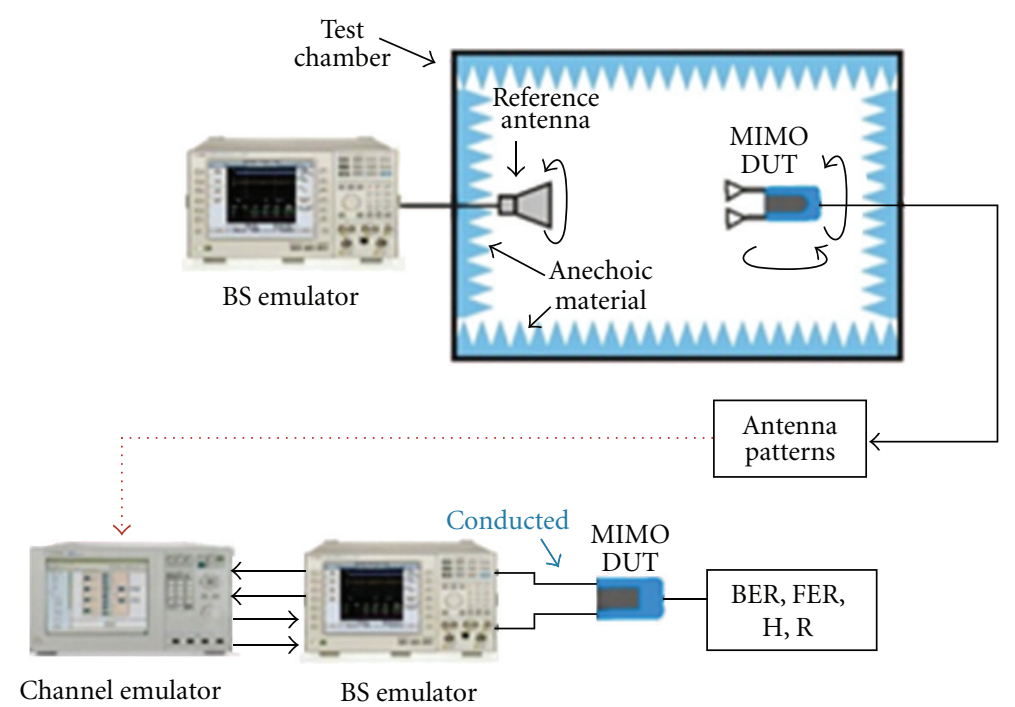

FIgURE 3: Proposed two-stage test methodology for MIMO OTA test (TR 37.976 [7] Figure 6.3.1.3.1-2).

conditions to test MIMO performance. It is illustrated in Figure 3. The first stage involves the measurement of the 3D antenna pattern of the DUT using an anechoic chamber of the size and type used for existing SISO tests. In order to measure the antenna pattern nonintrusively (i.e., without modification of the device or the attachment of cables), a special test function is required, which reports the received power per antenna and relative phase between antennas for a given received signal. The second stage takes the measured antenna pattern and convolves it with the desired channel model using a channel emulator. The output of the channel emulator then represents the faded downlink signal modified by the spatial properties of the DUT's antenna. This signal is then connected to the DUT's temporary antenna connectors as used for traditional conducted testing. The second stage does not require the use of an anechoic chamber.

The absolute accuracy of the DUT power measurement function is not critical since it is calibrated out as part of the second stage. The power linearity is more important but it too can also be linearized. The relative phase accuracy is important but this is an easier measurement for the DUT to make.

Since the 3D antenna pattern can easily be measured, the two-stage method can emulate any arbitrary 3D channel propagation condition. The rotation of the DUT relative to the channel model is accomplished by synthesis within the channel emulator. In its basic form where the antenna pattern is measured at a power well above reference sensitivity, a characteristic of the two-stage method is that the impact of self-interference is not captured.

Since spatial multiplexing requires relatively good SINR in order to provide gain, the spatial multiplexing performance at low signal levels is unlikely to be of significance. Standards exist for measuring SISO self-interference, and a study is underway to extend these simpler test systems for SIMO operation. However, since self-interference is included in the other MIMO candidate methodologies, work

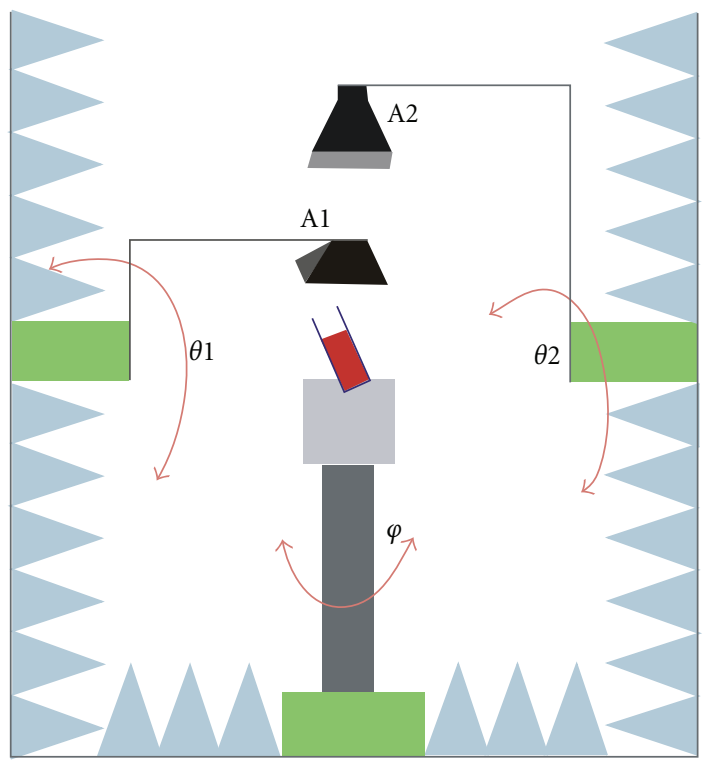

FIGURE 4: Two-Channel Method, antenna arrangement in anechoic chamber (TR 37.976 [7] Figure 6.3.1.4.1-1).

is underway to extend the two-stage method to include the evaluation of self-interference.

3.4. A4 Two-Channel Method. The two-channel method shown in Figure 4 is a special case of the multiprobe method and uses just two probes with no channel emulator. The angle of departure of the two downlink test signals can be configured for any azimuth, elevation, or polarization. The principle of the method is to evaluate the impact of the direction and angular separation of the two signals on the DUT performance. By carrying out a large number of tests using different combinations of angles, statistical analysis can be used to derive figures of merit for the DUT. Direct 


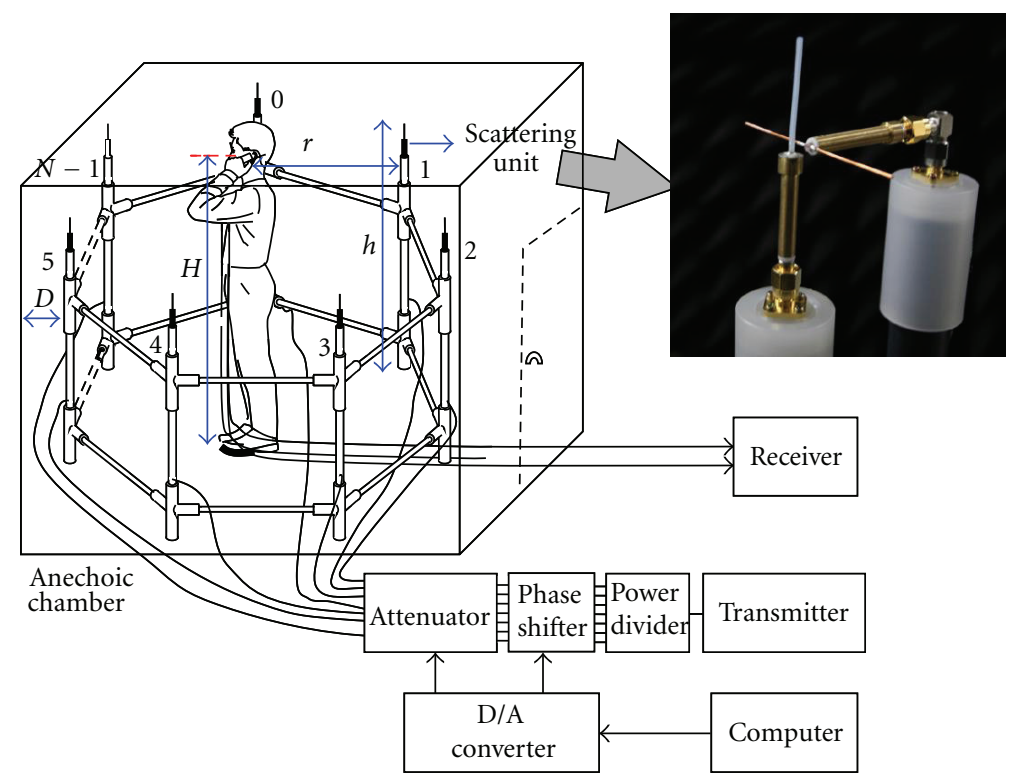

FIGURE 5: Experimental setup of the spatial fading emulator (TR 37.976 [7] Figure 6.3.1.5.1-1).

comparison with results achieved using more complex spatial signals with temporal variations is not possible, but results show this method to provide similar DUT ranking.

3.5. A5 Spatial Channel Emulation Method. The last of the anechoic chamber methods is a variation of the ring of probes method, where the channel emulation function is provided by a much simpler programmable attenuator and phase shifter per antenna. This is shown in Figure 5.

By controlling the amplitude and phase in real time, a Rayleigh distribution or other relevant multipath distribution can be obtained. This method generates an equal angular distribution for all propagation delays.

3.6. R1 Reverberation Chamber Method. The first of the reverberation-based methods uses the intrinsic reflective properties of the mode-stirred reverberation chamber to transform the downlink test signal into a rich 3D multipath signal. This is shown in Figure 6. The spatial characteristics of the signal are random and over time can be shown to be isotropic, but when observed over the time period of a demodulated data symbol, they are known to be highly directional. This nonuniformity provides the DUT with diverse signals on each antenna thus enabling spatial multiplexing gain.

The natural time domain response of the chamber can be modified by use of small amounts of RF absorptive material. The basic reverberation chamber is limited to a single power delay profile, and a relatively slow Doppler spectrum determined by the speed of the mode stirrer. Further control of the power delay profile and spatial aspects can be obtained by cascading two or more reverberation chambers as shown in Figure 7, and there has also been research by NIST and EMITE using nested chambers and coupled chambers.

In addition to the conventional Rayleigh 3D isotropic fading scenario emulated by single-cavity reverberation chambers, multicavity multisource mode-stirred reverberation chambers employ deembedding algorithms for enhanced repeatability and have added capabilities to emulate different K-factors for Rician fading, different nonisotropic scenarios including single and multiple-cluster with partial door opening, and standardized or arbitrary amplitude power delay profiles (e.g., 802.11n, Nakagami-m, on-body and user-defined) using sample selection techniques.

\subsection{R2 Reverberation Chamber and Channel Emulator Meth-} od. The final method shown in Figure 8 addresses the limitation of the basic or cascaded reverberation chamber by adding a channel emulator to the downlink prior to launching the signals into the chamber. This allows the temporal aspects of the desired channel model to be fully controlled, although the underlying natural and very short decay time of the chamber will slightly spread the power delay profile.

With the use of a channel emulator capable of negative time delay (inverse injection), multiple cavity mode-stirred reverberation chambers can accurately emulate the power delay profiles of 3GPP SCME channel models.

\section{Comparison of Methods}

All seven methods have unique attributes, some of which are desirable and others less so. Section 9.1 of TR 37.976 provides an extensive list of these attributes. A simplified summary of the key points is given in Table 1 . This includes an assessment of the key technical areas still under study.

\section{Summary of Papers Accepted for Publication}

An unprecedented compilation of the latest research results for all methods can be found in this special issue. Twelve papers have been accepted for publication with an acceptance ratio below $32 \%$. 


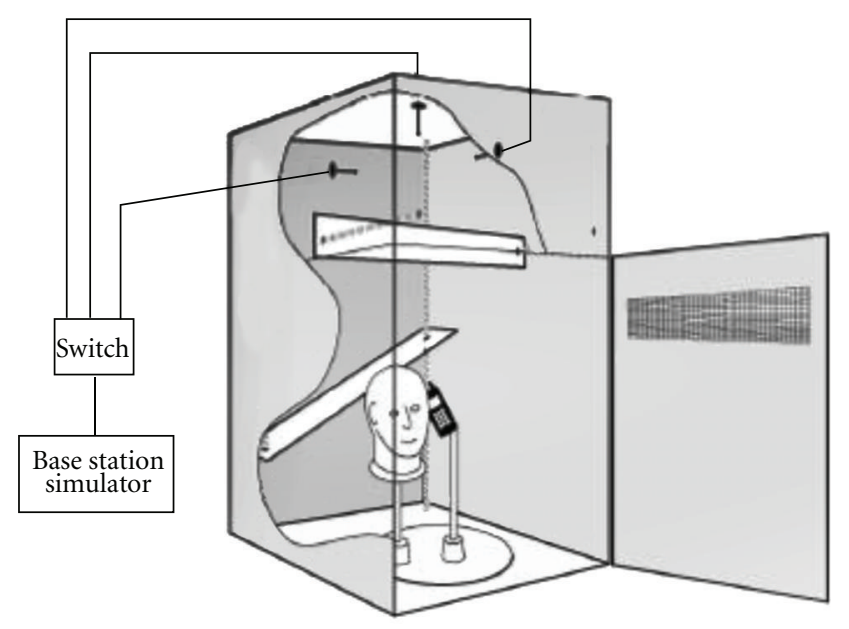

FIGURE 6: Reverberation chamber setup for devices testing with single cavity (TR 37.976 [7] Figure 6.3.2.1-1).

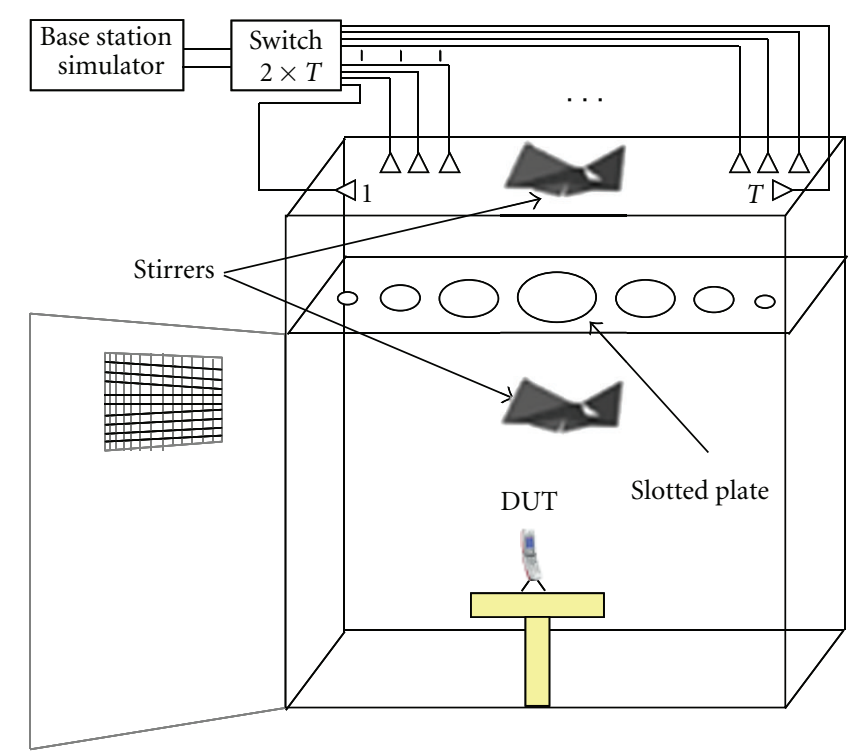

FIGURE 7: Reverberation chambers with multiple cavities (TR 37.976 [7] Figure 6.3.2.1-2).

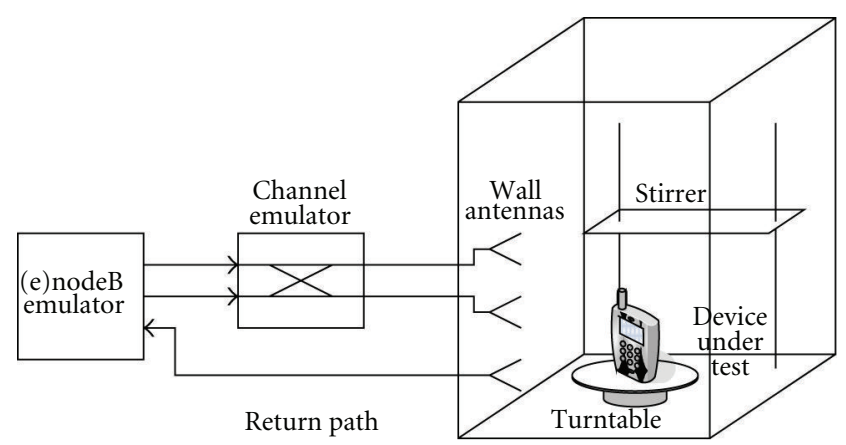

FIGURE 8: Test bench configuration for test using channel emulator and reverberation chamber for a $2 \times 2$ MIMO configuration TR 37.976 [7] Figure 6.3.2.2.1-1). 
TABLE 1: Comparison of candidate methodologies.

\begin{tabular}{llll}
\hline Method & Pros & Cons & Future work \\
\hline A1 Multiprobe & Conceptually simple & Limited flexibility Cost per probe & Calibration and validation \\
A2 Ring of probes & Arbitrarily flexible & Cost 3D very costly & Calibration and validation \\
A3 Two-stage & Low cost including 3D & Requires DUT test mode & Self-interference solution \\
A4 Two-channel & Very low cost & No temporal and limited spatial control & Correlation with other methods \\
A5 Spatial emulator & Low cost & Limitations in channel models & Calibration and correlation with other methods \\
R1 Reverb & Very low cost & Limited temporal and no spatial control & Calibration and evaluation of spatial aspects \\
R2 Reverb plus fader & Low cost & No spatial control & Calibration and evaluation of spatial aspects \\
\hline
\end{tabular}

Among the methodology-agnostic contributions, the work by Kanemiyo et al. in [8] highlights the still-existing differences between realistic fading channels and simplified channel models. A new channel model based on correlation with a given fixed theoretical correlation between antenna elements at the mobile is provided. This MIMO channel model can be used for studying the relationship between the correlation and eigenvalues for various propagation environments. These differences are also dealt with in the work by Nguyen et al. [9], wherein a specific channel model is derived using the promising time-reversal technique. By using TimeReversal (TR), several data streams can be simultaneously transmitted by using only one antenna while outperforming a true MIMO-UWB (Ultra WideBand) system with multiple transmit antennas. The channel measurements are performed in a short-range indoor environment, using both line-of-sight and non-line-of-sight to verify the adopted correlated channel model. The interesting work by Pirkl and Remley [10] investigates the possibility of obtaining comparable throughput results between different test methodologies. In their work, it is demonstrated that, provided (1) the DUT is rotated to different orientations in the 2D statistically isotropic anechoic environment and (2) the dimensions of the DUT are on the order of a wavelength or less such that the element directivities will be low, we can expect that throughput statistics for a DUT in 2D and 3D statistically isotropic environments will be within $10 \%$ of each other. This suggests that test procedures for MIMO OTA wireless terminals in anechoic chamber (AC) and reverberation chamber (RC) methods should be comparable for the conditions studied.

Several papers provide interesting results for the RC methods. In [11], the joint research effort of SP in Sweden and UPCT in Spain show that it is possible for a multicavity RC to emulate different channel models with diverse levels of correlation using a novel sample-selection technique. The use of simple NIST channel models in a RC to emulate more complex channel models is an interesting method for standardisation. The 3GPP MIMO OTA Work Item in progress highlighted [12] a recent contribution by EMITE in which "New figures of merit were presented which seem to be a very useful tool in order to analyze the large amount of information that will be available once a certain or set of methods are found to provide meaningful and comparable results." The new figures of merit, which are a statistical analysis of measured throughput, are presented in this issue by Marin-Soler et al.
[13]. These figures can indeed be very useful for determining the final goal of distinguishing between good and bad MIMO devices with a large set of measured throughput data obtained for a specific device following the 3GPP/CTIA test plans. The differences between test methods observed during measurement campaigns can be mitigated for RCs by the novel calibration method presented by García-Fernández et al. [14]. The new calibration method can provide a prediction of the field uniformity mean value from just one field amplitude measurement, taking advantage from the statistical laws that describe electromagnetic field distribution behavior, thus saving more than $95 \%$ of the calibration time and reducing realization costs. The ability of RCs to emulate the time domain aspects of 3GPP SCME channel models is demonstrated by Arsalane et al. [15]. In their work, a multicluster channel with the same delay spread for each cluster is emulated using a $\mathrm{RC}$ by convolving the base band signal to be transmitted with the urban macrocell (UMa) or urban microcell (UMi) channel model tap delay line generated using a MATLAB program. The obtained Power Delay Profiles (PDPs) are verified by channel sounding based on a sliding correlation and show very good agreement to the theoretical 3GPP SCME UMi and UMa channel models. The work by Hansen [16] concentrates on demonstrating the ability of RCs to evaluate antenna correlations and to match the results obtained in an isotropic environment to those obtained from the classical definition. Clearly distinguishable capacity curves are also provided for the CTIA-approved good, nominal, and bad reference antennas.

The contributions related to ACs are equally interesting. Khatun et al. [17] clarify the very important and cost-related issue of the required number of probes for synthesizing the desired fields inside a multiprobe system. Rules are presented for the required number of probes as a function of the test zone size in wavelengths for certain chosen uncertainty levels of the 2D field synthesis in an AC. The work by Kyosti et al. [18] show that the creation of a propagation environment inside an $\mathrm{AC}$ with the ring of probes method requires unconventional radio channel modelling, namely, a specific mapping of the original models onto the probe antennas, with the geometric description being a prerequisite for the original channel model.

For the Two-Channel method, the works by Feng et al. [19] show that this method is well suited to distinguish good and bad devices using two new statistical figures of merit and 
different realizations of two antennas in a distributed axis AC. Finally, the work by Jing et al. [20] describes the TwoStage method in detail. This method takes a fundamentally different approach to the problem. Unlike all the other methods, which attempt to create some form of spatial channel model into which the device is placed for measurement, the two-stage method instead measures the $3 \mathrm{D}$ antenna pattern of the device in a traditional SISO AC and then convolves the antenna pattern inside a channel emulator in order to make throughput measurements using cabled connections to the DUT's temporary antenna ports. The conducted signal received by the DUT thus emulates what would have been received by the DUT had it been placed in the radio field created by the channel emulator. The orientation of the DUT relative to the channel model is changed synthetically within the channel emulator, and the time-consuming throughput measurements do not require use of an AC.

\section{Future Work}

In addition to the method-specific open issues briefly summarized in Table 1, CTIA, and 3GPP are collaborating on future studies in order to evaluate the most appropriate radio conditions in which to measure MIMO OTA performance as well as elaborating and evaluating the capabilities of the candidate methodologies to differentiate good and bad MIMO devices. The newly approved MIMO OTA work item in 3GPP [6] is targeting December 2012 for completion.

A key element of these works is the development of reference antennas by CTIA, which will be used both in simulation of expected performance in known channel conditions and actual measurements on real devices. These steps will provide the essential traceability required to finalize the development of conformance test methods and possible device minimum performance requirements. CTIA is also working on verification procedures to align the many environmental conditions that need to be controlled if measurements made using different equipment and methods are to be comparable. This effort will certainly minimise uncertainties in the final results provided by different MIMO OTA test methods. Furthermore, the use of the reference antennas, selected reference channel models, and other environmental considerations will provide important information required by 3GPP/CTIA to make a final decision for the selected standardised test methods.

\section{Moray Rumney Ryan Pirkl Markus Hermann Landmann David A. Sanchez-Hernandez}

\section{References}

[1] European Cooperation in Science and Technology Action 259, "Wireless flexible personal communications," http://www .lx.it.pt/cost259/.

[2] European Cooperation in Science and Technology Action 273, "Towards mobile broadband multimedia networks," http:// www.cost.eu/domains_actions/ict/Actions/273.
[3] CTIA ERP, Test Plan for Mobile Station Over the Air Performance v1.0, October 2001.

[4] 3GPP, "Measurements of radio performances for UMTS terminals in speech mode," Tech. Rep. 25.914, 2006, v.7.0.0.

[5] 3GPP, "Measurement of radiated performance for MIMO and multi-antenna reception for HSPA and LTE terminals," RP090352, 2011.

[6] 3GPP, "Verification of radiated multi-antenna reception performance of UEs in LTE/UMTS," RP-120368, 2012.

[7] 3GPP, "Measurements of radiated performance for MIMO and multi-antenna reception for HSPA and LTE terminals," Tech. Rep. 37.976, 2012, v11.0.0.

[8] Y. Kanemiyo, Y. Tsukamoto, H. Nakabayashi, and S. Kozono, "MIMO channel model with propagation mechanism and the properties of correlation and eigenvalue in mobile environments," Hindawi IJAP Special Issue on MIMO OTA, 2012.

[9] H. Nguyen, V. D. Nguyen, T. K. Nguyen et al., "On the performance of the time reversal SM-MIMO-UWB system on correlated channels," Hindawi IJAP Special Issue on MIMO OTA, 2012.

[10] R. Pirkl and K. A. Remley, "MIMO channel capacity in 2-D and 3-D isotropic environments," Hindawi IJAP Special Issue on MIMO OTA, 2012.

[11] P. Hallbjorner, J. D. Sánchez-Heredia, and M. Antonio, "Mode-stirred chamber sample selection technique applied to antenna correlation coefficient," Hindawi IJAP Special Issue on MIMO OTA, 2012.

[12] 3GPP, "MIMO OTA way forward," R4-122132, March 2012.

[13] A. Marin-Soler, G. Ypina-Garcia, A. Belda-Sanchiz et al., "MIMO throughput effectiveness for basic MIMO OTA compliance testing," Hindawi IJAP Special Issue on MIMO OTA, 2012.

[14] M. A. García-Fernández, C. Decroze, D. Carsenat et al., "On the relationship between the distribution of the field amplitude, its maxima, and field uniformity inside a modestirred reverberation chamber," Hindawi IJAP Special Issue on MIMO OTA, 2012.

[15] N. Arsalane, M. Mouhamadou, C. Decroze et al., "3GPP channel model emulation with analysis of MIMO-LTE performances in reverberation chamber," Hindawi IJAP Special Issue on MIMO OTA, 2012.

[16] T. B. Hansen, "Correlation and capacity calculations with reference antennas in an isotropic environment," Hindawi IJAP Special Issue on MIMO OTA, 2012.

[17] A. Khatun, T. Laitinen, V. M. Kolmonen et al., "Dependence of error level on the number of probes in over-the-air multiprobe test systems," Hindawi IJAP Special Issue on MIMO OTA, 2012.

[18] P. Kyosti, T. Jämsä, and J. P. Nuutinen, "Channel modelling for multiprobe over-the-air MIMO testing," Hindawi IJAP Special Issue on MIMO OTA, 2012.

[19] Y. Feng, W. L. Schroeder, C. von Gagern et al., "Metrics and methods for evaluation of over-the-air performance of MIMO user equipment," Hindawi IJAP Special Issue on MIMO OTA, 2012.

[20] Y. Jing, X. Zhao, H. W. Kong et al., "Two-stage over the air (OTA) test method for LTE MIMO device performance evaluation," Hindawi IJAP Special Issue on MIMO OTA, 2012. 

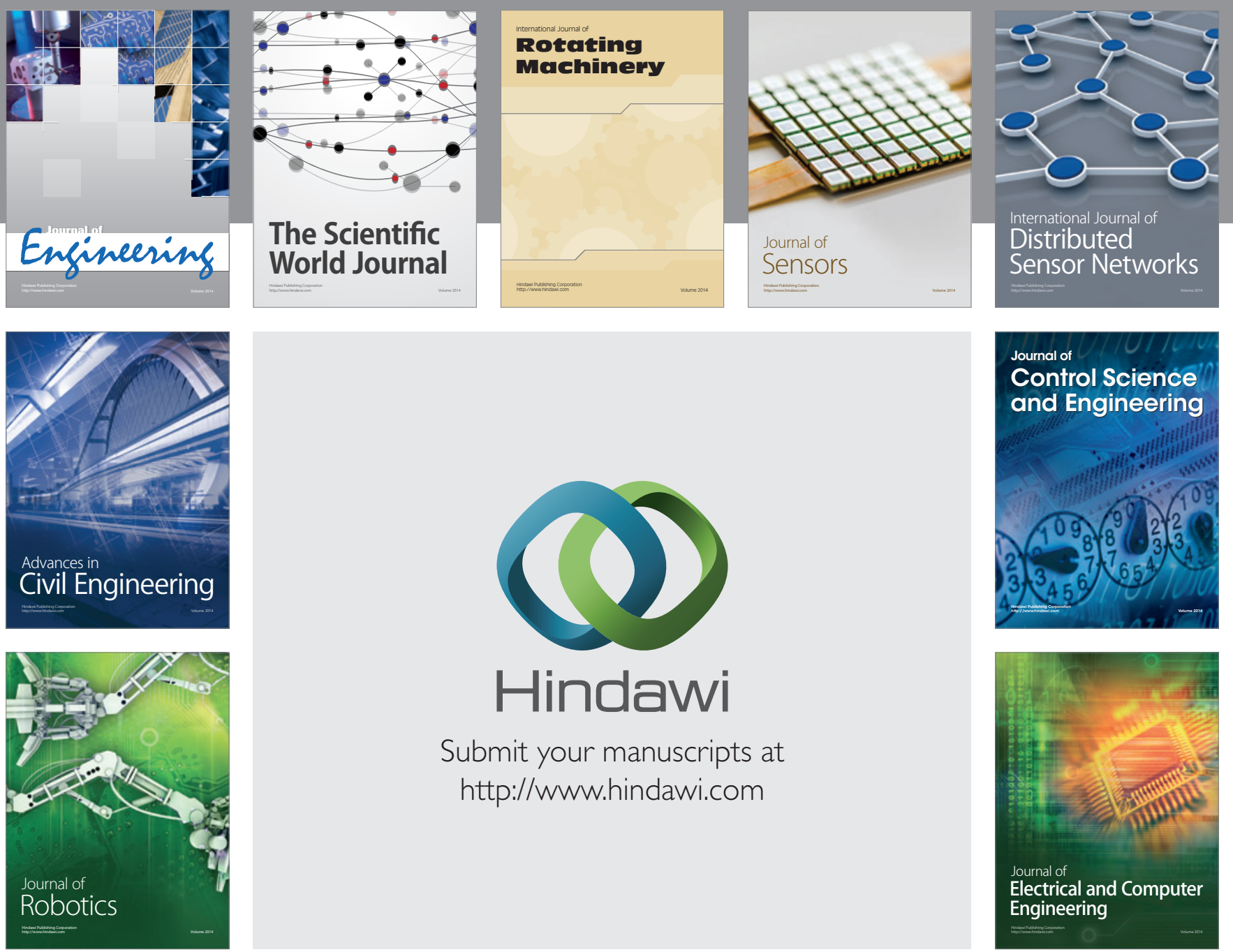

Submit your manuscripts at

http://www.hindawi.com
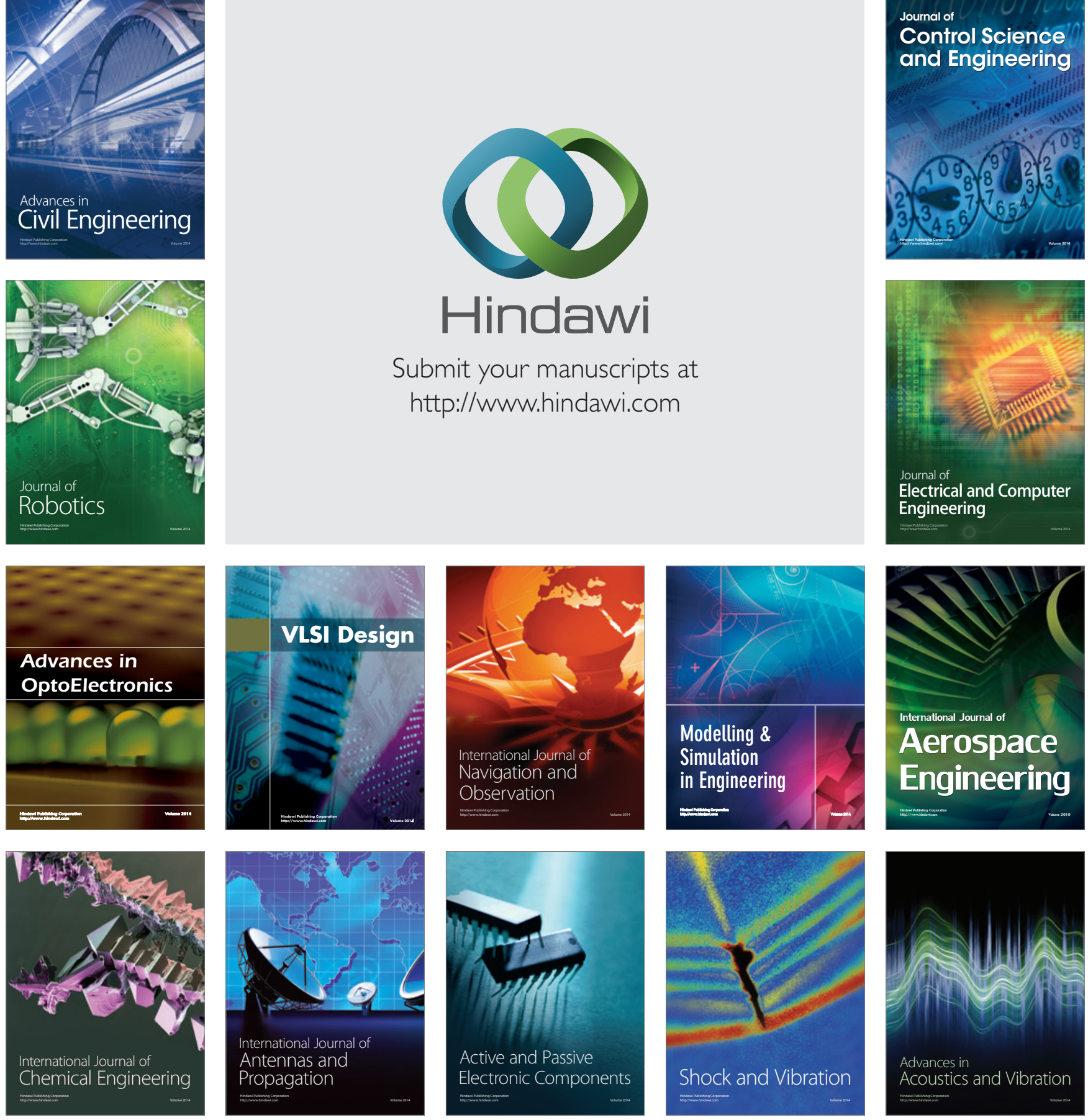